\title{
A comparative experimental kinetic study of spontaneous and plasma-assisted cool flames in a rapid compression machine
}

G. Vanhove ${ }^{\mathrm{a},{ }^{*}}$, M.-A. Boumehdi ${ }^{\mathrm{a}}$, S. Shcherbanev ${ }^{\mathrm{b}}$, Y. Fenard ${ }^{\mathrm{a}}$, P. Desgroux ${ }^{\mathrm{a}}$, S. M. Starikovskaia ${ }^{\mathrm{b}}$

a Univ. Lille, CNRS, UMR 8522 - PC2A - Physicochimie des Processus de Combustion et de l'Atmosphère, F-59000 Lille, France

b Laboratory of Plasma Physics (CNRS, Ecole Polytechnique, Sorbonne Universities, University of Pierre and Marie Curie - Paris 6, University Paris-Sud), Ecole Polytechnique, Route de Saclay, 91128 Palaiseau, France

* Corresponding author: PC2A - Université de Lille 1, Bâtiment C11, Cité scientifique, 59655 Villeneuve d'ascq cedex, France.

E-mail address: guillaume.vanhove@univ-lille1.fr (G. Vanhove) 


\begin{abstract}
Plasma-assisted cool flames of $n$-heptane were generated in the combustion chamber of a rapid compression machine coupled with a nanosecond dielectric barrier discharge, at a pressure of 1.5 bar and temperature $T_{C}=650 \mathrm{~K}$. Increasing of the voltage pulse amplitude at the electrode resulted in a transition from no reactivity to induced cool flame and then to fast ignition. Sampling of the reacting mixture was performed at selected times during the experiments to draw mole fraction profiles of the fuel and major low temperature stable intermediates, showing a gradual increase in the mole fraction of these species after the discharge. Comparison with a spontaneous cool flame case at a slightly higher pressure shows that no new species are formed in the plasma-assisted case, and that after the initiation of reactivity by the discharge at the nanosecond timescale, the distribution and relative importance of the main reaction pathways is conserved at the millisecond timescale. Differences in the shape of the mole fraction and light emission profiles however suggest that the plasma-assisted cool flame is propagative.
\end{abstract}

Keywords: Plasma-assisted ignition; low temperature combustion; cool flame; rapid compression machine; kinetics 


\section{Introduction}

In new engine technologies, such as PPCI (Partially Premixed Compression Ignition) or HCCI (Homogeneous Charge Compression Ignition) configurations [1], the initiation of combustion is highly dependent on the chemical kinetics associated with Low Temperature Combustion (LTC). This combustion regime is associated with degenerate chemical branching through the formation of highly unstable peroxidic species, the formation of such being constrained by the structure of the initial fuel [2]. This chemical branching results in cool flames, i.e. faint light emission around $400 \mathrm{~nm}$, usually attributed to the formation of excited formaldehyde, as well as a moderate temperature increase. Difficulties in ensuring reproducible ignition timing in such engine configurations has triggered interest in plasma-assisted ignition in the LTC regime [3-5].

Among the fuels that have been studied in such conditions, $n$-heptane is a good example, as it shows high LTC reactivity, is one of the Primary Reference Fuels for octane number measurement, and is often used as a surrogate for diesel fuel. Its LTC chemical kinetics have been studied in several reactors, including jet-stirred reactors [6,7], shock tubes [8] or rapid compression machines $[9,10]$. It is well established that the chain branching mechanism responsible for its LTC reactivity proceeds through the following steps:

- Initiation by hydrogen abstraction by $\mathrm{O}_{2}, \dot{\mathrm{O}} \mathrm{H}$ or HOÓ, yielding an $n$-heptyl radical and $\mathrm{HOO}, \mathrm{H}_{2} \mathrm{O}$ or $\mathrm{H}_{2} \mathrm{O}_{2}$ respectively,

- Addition of the $n$-heptyl radical to $\mathrm{O}_{2}$ molecules to form a $\mathrm{RO}_{2}$ radical,

- Internal isomerization of this $\mathrm{RO}_{2} \cdot$ into a ${ }^{\circ} \mathrm{QO}_{2} \mathrm{H}$ radical,

- Addition of the $\mathrm{QO}_{2} \mathrm{H}$ radical to $\mathrm{O}_{2}$ molecules,

- Formation and decomposition of ketohydroperoxides, yielding two ÖH radicals.

The thereby formed $\dot{\mathrm{OH}}$ radicals will take part in the initiation reactions and accelerate the global reactivity, resulting in chemical branching. In the reaction sequence that follows initiation, internal 
isomerization reactions are rate-limiting, making it more likely for species with a long alkyl chain. Other

LTC chemistry pathways consist in propagation reactions and yield stable intermediates, for example by decomposition of ${ }^{2} \mathrm{QO}_{2} \mathrm{H}$ radicals into alkenes, carbonyls and cyclic ethers, depending on the positions of the initial $\mathrm{H}$ abstraction and the internal isomerization.

The possibility to induce ignition of methane or $n$-butane in high pressure conditions in a Rapid Compression Machine (RCM), by means of local production of excited species and radicals by a Dielectric Barrier Discharge (DBD), has been demonstrated in a recent paper [11]. This work focuses on using the same tools to stimulate the LTC reactivity of $n \_$heptane.

\section{Experimental}

The coupling of the Lille University RCM [12] and the Laboratoire de Physique des Plasmas (LPP) DBD electrode system [13] has been described previously [11], so only the major elements will be reminded here. The RCM has right-angle geometry to ensure constant volume at Top Dead Center (TDC). During a classical experiment, the piston position, pressure and light emission are recorded, using respectively a Honeywell HOA 20001-001 optocoupler, a Kistler 6052 piezoelectric transducer coupled to a Kistler 5017 charge amplifier, and a RCA 1P21 photomultiplier. The initial temperature of the combustion chamber is fixed with a $\pm 1 \mathrm{~K}$ accuracy along the RCM axis. The temperature of the adiabatic core is calculated following the isentropic law from the initial temperature, initial and compressed pressures following the adiabatic core assumption [12]. The RCM is equipped with a creviced piston following the design suggested in [14] to limit mixing of the boundary layer with the adiabatic core. $99.8 \%$ grade $n$-heptane from Sigma-Aldrich and oxygen and nitrogen with $99.99 \%$ purity from Air Liquide were used to prepare gas mixtures in a separated facility, where they were left to homogenize overnight. 
The DBD electrode system was installed in the place of the end plate of the RCM. It consists of a central coaxial high voltage (HV) electrode which is ended by a brass convex segment of a radius of a curvature of $50 \mathrm{~mm}$. The outer diameter of the segment is $20 \mathrm{~mm}$ and equal to the inner diameter of the low voltage grounded electrode. The low voltage electrode is made of aluminum. The high and low voltage electrodes are separated with a thin, $0.3 \mathrm{~mm}$, dielectric PVC layer. A FID Technology FPG20-03NM high voltage generator delivered single pulses of negative or positive polarity, $12-55 \mathrm{kV}$ in amplitude, 2 ns pulse front rise time and $20 \mathrm{~ns}$ pulse duration on the half-height to the electrode through a $30 \mathrm{~m}$ coaxial high-frequency RG213 cable. Two calibrated custom-made back current shunts (BCS) were installed in the cable shield break at half the length of this cable and $1 \mathrm{~m}$ before the electrode. The first BCS was used to measure the waveforms of the incident pulse of electrical current/voltage and of the pulse reflected from the electrode system. The second was used for synchronization of RCM and the high-voltage generator. A LeCroy Wave Runner $600 \mathrm{MHz}$ oscilloscope was used to monitor the signals, which can later be used to extract the energy deposited in the discharge. The absence of spark formation was controlled from current waveforms for all the experiments presented here.

To probe the formation of stable oxidation intermediates during the experiments, the whole contents of the reaction chamber were extracted in a separate vessel, as applied previously in autoignition experiments [15], the pressure drop being fast enough to quench the reactivity in less than a millisecond. Acquired samples were analyzed offline in a Bruker Scion Gas Chromatograph equipped with mass spectrometry and FID/TCD detection. The detected intermediates were identified on the basis of their mass spectra. Calibration of the detectors was performed for the species that are readily available, and estimated by the effective carbon number otherwise, resulting in a $10 \%$ estimated relative error on the mole fractions. 


\section{Discharge development}

To investigate the effect of the pressure on the discharge morphology, spectrally integrated (300$800 \mathrm{~nm}$ ) images of discharge were recorded in a stoichiometric $n$-heptane/synthetic air mixture using a Princeton Instruments PI-MAX 2 ICCD (RB Fast Gate Gen 2) camera through the side window of the RCM, as shown in Figure 1. Spectral analysis showed that light emission corresponds to transitions relative to excited $\mathrm{N}_{2}$ exclusively. It was found in [11] that at high pressures the discharge develops mainly in the plane of the electrode system. At low pressures the discharge is volumetric: longitudinal structures can be seen at the left hand side of Figure 1. The energy delivered to the discharge does not exceed (12-17) $\mathrm{mJ}$ at $\mathrm{P}_{\mathrm{TDC}}=1.5$ bar. This corresponds to typical values in a DBD without or with partial closing of the electric gap, we will therefore refer to these longitudinal structures as streamers in the rest of this study. Figure 1 shows that the number of streamers is superior in the negative polarity case.

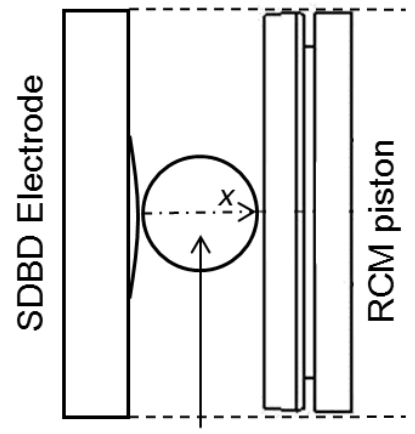

Side window
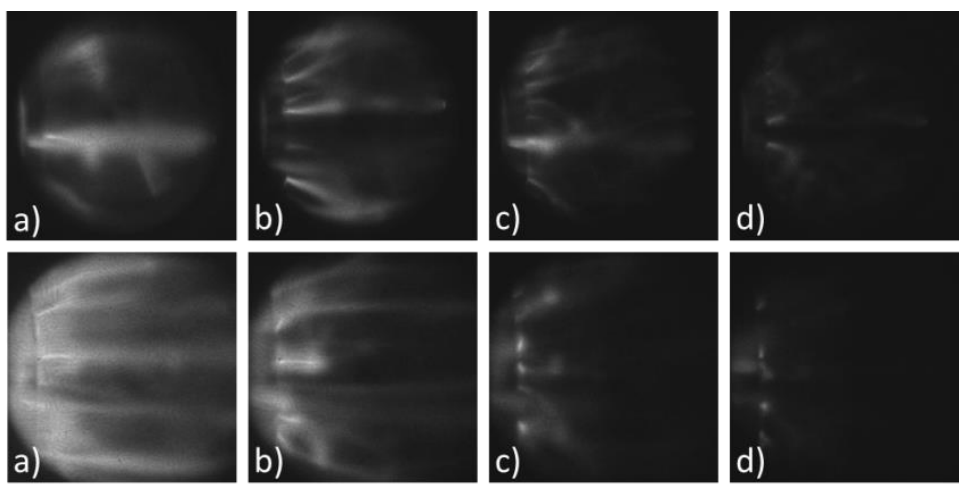

Figure 1: Side imaging of the discharge development in the RCM. $n-\mathrm{C}_{7} \mathrm{H}_{16} / \mathrm{O}_{2} / \mathrm{N}_{2}$ mixture, $\mathrm{T}_{\mathrm{C}}=650 \mathrm{~K}$. Upper row: $\mathrm{U}=50 \mathrm{kV}$; Lower row: $\mathrm{U}=-55 \mathrm{kV}$. a) $\mathrm{P}_{\mathrm{TDC}}=1.5$ bar b) $\mathrm{P}_{\mathrm{TDC}}=2.1$ bar c) $\mathrm{P}_{\mathrm{TDC}}=2.7$ bar d)

$$
\mathrm{P}_{\mathrm{TDC}}=3.3 \text { bar. }
$$

The discharge characteristics were investigated further by means of emission spectroscopy. An Acton Research 300i imaging spectrometer equipped with a 1200 gr.mm ${ }^{-1}$ grating was used in conjunction with the ICCD camera to record 1D-spectral images of the discharge. Figure 2 shows the evolution of the excited $\mathrm{N}_{2}$ emission spectrum as a function of the distance from the electrode ( $\mathrm{x}$ axis in Figure 1). In 
such images, the recorded intensities correspond to a spatially integrated value along the depth of the combustion chamber. A binned spectrum, not corrected for the transmission function of the detection system, is also presented in Figure 2. This variation of transmission is considered negligible between 350 and $360 \mathrm{~nm}$, so that the two transitions $\left(v^{\prime}=1 \rightarrow v^{\prime \prime}=2\right)$ at $353 \mathrm{~nm}$ and $\left(v^{\prime}=0 \rightarrow v^{\prime \prime}=1\right)$ at $357 \mathrm{~nm}$ can be used to evaluate the vibrational temperature $\mathrm{T}_{\mathrm{vib}}$. Note that this evaluated value of $\mathrm{T}_{\mathrm{vib}}$ corresponds to the vibrational temperature of a homogeneous discharge, which could differ from our experimental case. Figure 2 also displays several fits of the normalized spectrum in the region 345-365 $\mathrm{nm}$ as a function of $\mathrm{T}_{\text {vib}}$, obtained using the SPECAIR software [16]. In these calculated spectra, the electronic and rotational temperatures were fixed respectively to $12000 \mathrm{~K}$ and $650 \mathrm{~K}$, both being rather non-sensitive parameters given the relatively low resolution of the spectrometer. From these fits the equivalent $\mathrm{T}_{\mathrm{vib}}$ for a homogeneous discharge can be evaluated to approximately (3500 \pm 500$)$ K. In images such as Figure 2 , the ratio of the peak intensities of the transitions $\left(\mathrm{v}^{\prime}=0, \mathrm{v}^{\prime \prime}=1\right)$ and $\left(\mathrm{v}^{\prime}=1, \mathrm{v}^{\prime \prime}=2\right)$ is therefore correlated to this equivalent $\mathrm{T}_{\mathrm{vib}}$. An investigation of the evolution of the ratio of the peak intensities of these two bands, evaluated in 6 regions equally spaced along the $\mathrm{x}$ axis, showed that there is no significant evolution of this ratio along the length of the streamers, within the uncertainty limits.

\section{Plasma assisted cool flames}

Previous work at higher pressures [11] showed that when the amplitude of the voltage pulse increased, an abrupt transition was observed between a situation where the ignition delay was identical to the autoignition case to a situation where the ignition delay was reduced by more than an order of magnitude. Following plasma-assisted ignition, a hot flame front propagated from the electrode towards the piston, with a velocity that was not affected by the discharge. 


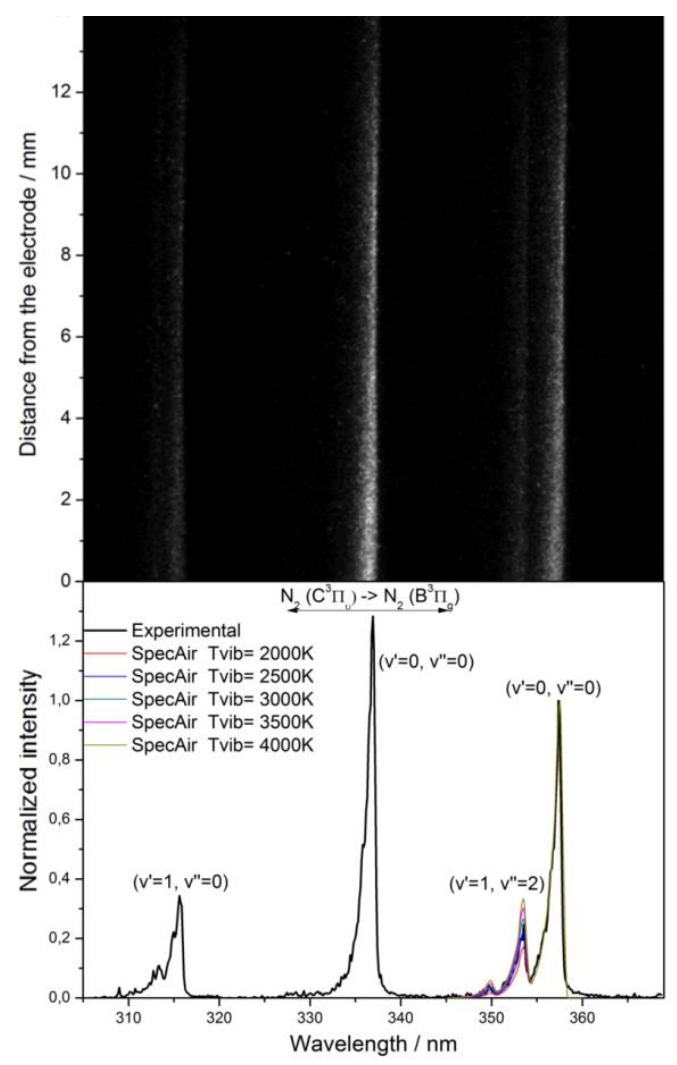

Figure 2: 1D-spectral images of the discharge along the $\mathrm{x}$ axis shown in Figure 1 and corresponding binned spectrum. $\mathrm{T}_{\mathrm{C}}=650 \mathrm{~K}, \mathrm{P}_{\mathrm{TDC}}=1.5$ bar, $\mathrm{U}=-(35 \pm 2) \mathrm{kV}$.

In lower pressure conditions of $\mathrm{P}_{\mathrm{TDC}}=1.5$ bar and $\mathrm{T}_{\mathrm{C}}=650 \mathrm{~K}$, using $n$-heptane as the fuel and with a pulse of negative polarity, a case where a cool flame is induced by the discharge has been identified, as displayed in Figure 3. Without discharge or with the lowest values of the absolute value of the voltage amplitude $|\mathrm{U}|$, the system showed no reactivity. For intermediate values of $|\mathrm{U}|$, the system displayed a cool flame, as visible from the limited pressure increase and light emission detected by the photomultiplier. At higher voltages hot ignition was observed, as in [11]. The absence of reactivity without plasma was verified, ensuring reproducibility of this situation. In the case of positive polarity pulses, no plasma-assisted cool flame was observed as the system evolved directly from no reactivity to ignition. 


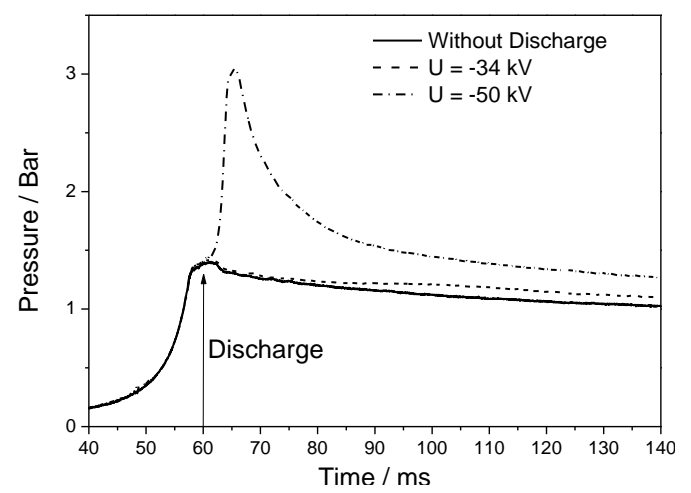

Figure 3: Effect of the voltage pulse amplitude on the pressure profile during plasma-assisted ignition experiments. Stoichiometric $n$-heptane/synthetic air mixtures, $\mathrm{T}_{\mathrm{C}}=650 \mathrm{~K}$.

To gain insight on possible modifications by the plasma of the main reaction pathways associated with $n$-heptane oxidation in low temperature combustion conditions, the reacting mixture was quenched at selected times after the discharge in separate experiments, and analyzed using gas chromatography techniques. For this series of experiments, the pulse voltage on the high-voltage electrode was ($35 \pm 2) \mathrm{kV}$. It should be noted that no significant modification of the discharge morphology was observed when the voltage was varied from -35 to $-55 \mathrm{kV}$. The major intermediate products formed in $n$-heptane cool flames, as reported in [9], were detected. These include $\mathrm{C}_{7}$ cylic ethers, alkenes, and ketones, such as 5-ethyl,2-methyltetrahydrofuran, 2-propyltetrahydrofuran, 1- and 2-heptene, and 3-heptanone, $\mathrm{C}_{2}$ to $\mathrm{C}_{6}$ 1-alkenes, and small oxygenates like butanal, butanone, oxetane, propanal, and acetaldehyde. Traces of methanol, water, and acetone were detected but not quantified. 

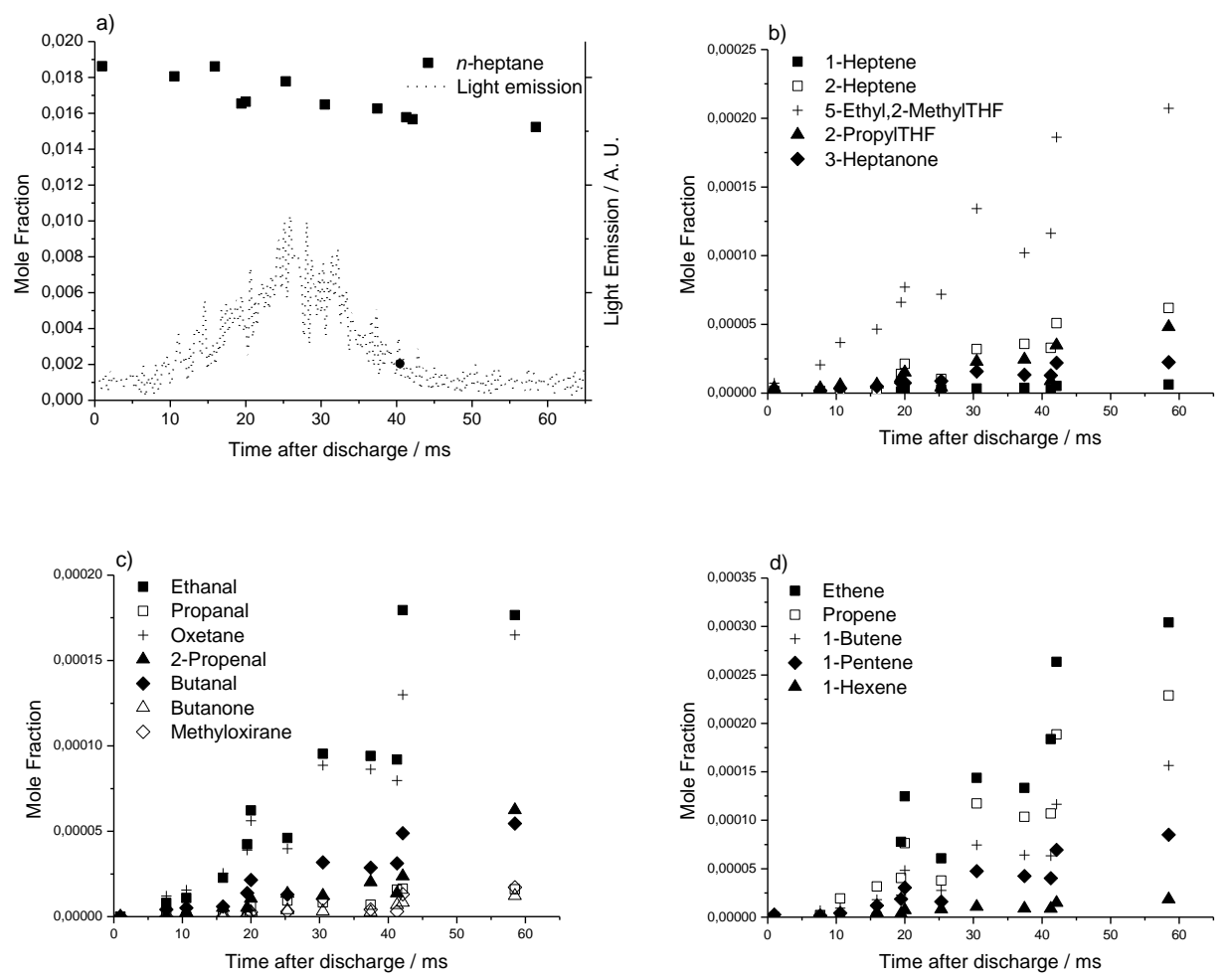

Figure 4: Mole fraction profiles of the major species formed during the plasma-assisted cool flame of a stoichiometric $n$-heptane/synthetic air mixture. $\mathrm{P}_{\mathrm{TDC}}=1.5 \mathrm{bar}, \mathrm{T}_{\mathrm{C}}=650 \mathrm{~K}$.

Mole fraction profiles after the discharge were drawn and are presented in Figure 4. The apparent scatter in the reported mole fraction data can partly be attributed in the small variation of the amplitude of the voltage pulse at the electrode between each of the sampling experiments. This resulted in variability in the intensity of the cool flame. Figure 4a) shows the evolution of the mole fraction of $n$-heptane after the discharge, as well as the photomultiplier signal in such experiments. The consumption of $n$-heptane in the cool flame is approximately $15 \%$. The decrease in $n$-heptane mole fraction is gradual with time, and the light emission by the cool flame is faint and has a duration of more than $30 \mathrm{~ms}$. Among $\mathrm{C}_{7}$ species, the two isomers of 5-ethyl,2-methyltetrahydrofuran are the species formed in the highest quantities, as visible on Figure 4b). Trace quantities of this species and 2-propyltetrahydrofuran are visible at very early times after the discharge. Figure 4c) shows that ethanal and oxetane are the major small 
oxygenates. Figure 4d) presents the mole fraction profiles of 1-alkenes: these species are formed in increasing quantities as their number of $\mathrm{C}$ atoms decreases, as is usually observed in this case.

These analyses were compared to results obtained in a spontaneous cool flame, at a slightly higher pressure of 2.4 bar, same temperature of $650 \mathrm{~K}$, and without plasma. It should be noted that in both cases only the cool flame was observed, and that there was no second stage, or high temperature ignition, as can be observed in higher pressure cases [17]. This is possibly due to a lower reaction rate for $\mathrm{H}_{2} \mathrm{O}_{2}+\mathrm{M} \rightarrow 2 \cdot \mathrm{OH}+\mathrm{M}$ associated with these lower pressure cases, since the competition between this reaction and heat losses to the walls plays an important role in the possibility of ignition in this temperature regime [17].

Figure 5 displays chromatograms acquired in the two investigated cases. It is clearly visible that no new product was found in the case of the plasma-assisted cool flame. This was verified at all sampling times. A comparison of the mole fraction profiles for 5-ethyl,2-methyl tetrahydrofuran, one of the major stable intermediates formed in both cool flame, is displayed in Figure 6. While mole fraction profiles are shown here for only one species for the sake of brevity, it should be noted that the conclusions drawn here remain true for other products. A raw comparison of these concentration profiles shows that in the case of the plasma-assisted cool flame, the formation of products starts right after the discharge, and is more gradual than in the spontaneous cool flame case. In the latter, intermediates are formed readily at the cool flame delay of approximately $25 \mathrm{~ms}$. 


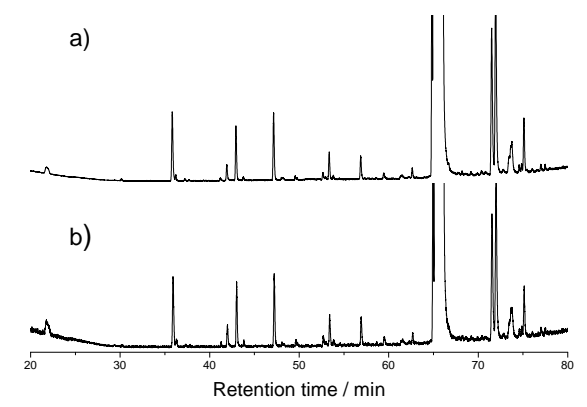

Figure 5: Chromatograms obtained during the analyses of samples withdrawn after a) A plasma-assisted cool flame, with $\mathrm{T}_{\mathrm{C}}=650 \mathrm{~K}$, and $\mathrm{P}_{\mathrm{TDC}}=1.5$ bar; b) A spontaneous cool flame, with $\mathrm{T}_{\mathrm{C}}=650 \mathrm{~K}$ and

$$
\mathrm{P}_{\mathrm{TDC}}=2.4 \text { bar. }
$$

\section{Discussion}

At such relatively low pressures the thermal boundary layer can be thick in the RCM combustion chamber, and it is possible that mixing of this boundary layer with the adiabatic core reduces the size of said core despite the use of a creviced piston, as pointed out in [18]. Nevertheless, in the spontaneous cool flame case, the cool flame is expected to take place in the core gas region, where the gases are the hottest, especially as the core gas temperature is inferior to $700 \mathrm{~K}$, the temperature at which Negative Temperature Coefficient behavior is observable [9]. This is visible from the light emission profiles presented in Figure 6, where the light is emitted abruptly at the cool flame delay, in coherence with simultaneous ignition of the adiabatic core gas. In contrast, in the plasma-assisted cool flame case, light emission is more gradual and lasts for a longer time. These differences in the shape of the light emission and mole fraction profiles indicate that the plasma assisted cool flame is initiated at the location of the discharge streamers, and gradually propagates to the remaining of the combustion chamber. 


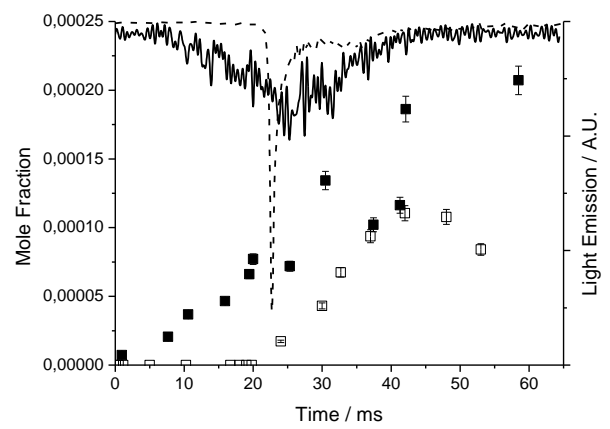

Figure 6: 5-Ethyl,2-methyltetrahydrofuran mole fraction (symbols) and light emission (lines) profiles after the discharge in the plasma-assisted cool flame case (full symbols and lines) and after the top dead center in the spontaneous cool flame case (open symbols, dashed line). The light emission profile for the plasma-assisted case is multiplied by 10 for clarity purposes.

To evaluate possible modification of the main reaction pathways by the discharge, the selectivity of the quantified products was calculated. Not all the products formed in the cool flame are quantified, and it is likely sensible quantities of $\mathrm{CO}$ and formaldehyde are formed during the cool flame, as observed previously for $n$-heptane [9] or other hydrocarbons [15]. The relative carbon selectivity was therefore calculated using the following equation:

$$
s_{i}=\frac{x_{i} \cdot n_{i}}{\sum_{j}^{\text {products }}\left(n_{j} \cdot x_{j}\right)}
$$

Where $x_{i}$ is the measured mole fraction and $n_{i}$ the number of carbon atoms in species $i$. $s_{i}$ therefore represents the proportion of carbon atoms quantified in the products that is present in species $i$.

Average values for $s_{i}$ after the beginning of the cool flame are presented in Table 1, along with their standard deviation calculated from all the samples acquired after the beginning of the cool flame. For all the quantified species, no significant difference in $s_{i}$ can be noted. This denotes that once the cool flame is initiated, the relative importance of the reaction pathways associated with the cool flame is respected. It is therefore likely that the discharge results in the generation of a pool of radicals through the dissociation of $\mathrm{O}_{2}$ molecules yielding atomic oxygen, as described in [4,5,11], but also through more 
direct interaction of the plasma with $n$-heptane molecules, this channel being however less described in the literature, or formation of NO which can react with HOO radicals to form $\dot{O} H$ radicals [5]. This radical pool consequently increases the reaction rates of initiation pathways and therefore the concentration of $n$-heptyl radicals, in accordance with the reaction mechanism summarized in the Introduction. The following reaction sequence responsible for the cool flame, of addition to $\mathrm{O}_{2}$ molecules, internal isomerization, second addition to $\mathrm{O}_{2}$, formation and decomposition of ketohydroperoxides [2], is in parallel less dependent on the concentration of small radicals. These processes indeed take place through unimolecular reactions or reactions with $\mathrm{O}_{2}$, which is readily available given its high concentration. The effect of the plasma on the reactivity can therefore be considered to originate mostly from an acceleration of the initiation reactions, with no significant modification of the main reaction pathways at the millisecond timescale. 
Species

Structure

Relative Carbon

Relative Carbon

selectivity

selectivity

Spontaneous Cool

Plasma-Assisted Cool

Flame

Flame

\begin{tabular}{|c|c|c|c|}
\hline Ethene & $=$ & $0.107 \pm 0.011$ & $0.097 \pm 0.014$ \\
\hline Propene & $=$ & $0.102 \pm 0.023$ & $0.097 \pm 0.012$ \\
\hline Ethanal & $\mathrm{O}=$ & $0.057 \pm 0.017$ & $0.059 \pm 0.004$ \\
\hline 1-Butene & & $0.087 \pm 0.011$ & $0.082 \pm 0.011$ \\
\hline Propanal & $\mathrm{O}=$ & $0.007 \pm 0.005$ & $0.009 \pm 0.006$ \\
\hline 2-Propenal & & $0.026 \pm 0.013$ & $0.017 \pm 0.007$ \\
\hline Oxetane & $c^{\circ}$ & $0.078 \pm 0.011$ & $0.077 \pm 0.003$ \\
\hline Methyloxirane & 只 & $0.008 \pm 0.004$ & $0.005 \pm 0.003$ \\
\hline 1-Pentene & & $0.068 \pm 0.013$ & $0.064 \pm 0.005$ \\
\hline Butanal & & $0.044 \pm 0.004$ & $0.044 \pm 0.002$ \\
\hline 1-Hexene & & $0.021 \pm 0.005$ & $0.019 \pm 0.005$ \\
\hline Butanone & L & $0.017 \pm 0.018$ & $0.006 \pm 0.002$ \\
\hline 1-Heptene & & $0.008 \pm 0.004$ & $0.007 \pm 0.002$ \\
\hline 2-Heptene & & $0.087 \pm 0.024$ & $0.080 \pm 0.012$ \\
\hline 5-Ethyl,2-methyltetrahydrofuran & & $0.216 \pm 0.055$ & $0.264 \pm 0.037$ \\
\hline 2-Propyltetrahydrofuran & & $0.040 \pm 0.006$ & $0.042 \pm 0.015$ \\
\hline 3-Heptanone & & $0.027 \pm 0.013$ & $0.030 \pm 0.006$ \\
\hline
\end{tabular}

Table 1: Species quantified during the plasma-assisted and spontaneous cool flame experiments and their relative carbon selectivity, as defined in the text, in both cases. 


\section{Conclusions}

Plasma-assisted cool flames were generated in a RCM at moderate pressures, by means of a nanosecond DBD discharge, and subject of a detailed experimental study by means of imaging, emission spectroscopy, and chemical analysis of the stable intermediate species. The results of this study show that under adequate pressure conditions, the DBD electrode system used in this study generates a volumetric discharge with streamers that cross the RCM chamber. The vibrational temperature along the streamers does not show significant variation along their length. Single-shot discharges enable initiation of the cool flame reactivity in an otherwise unreactive case. Comparison of these plasma-assisted cool flames with spontaneous cool flames obtained at a slightly higher pressure showed that no new products are formed. Differences in the shape of species and light emission profiles indicate that the observed plasma-assisted cool flames are propagative by nature, in contrast to the spontaneous cool flames which take place simultaneously in the whole combustion chamber. Quantification of the cool flame intermediates demonstrates that there is no discernible modification of the main reaction pathways associated to LTC chemistry, which leads to the conclusion that in this case, the effect of the discharge mainly results in the formation of a radical pool that accelerates the initiation steps of the LTC chemical mechanism. This indicates that the widespread modeling assumption of temporal decoupling between generation of active species and heat by the discharge and combustion phase is applicable in this case.

\section{Acknowledgements}

This work was funded by the ANR Project PLASMAFLAME, 2011 BS09 02501, and is a contribution to the CPER research project CLIMIBIO. The authors thank the French Ministère de l'Enseignement Supérieur et de la Recherche, the Hauts de France Region and the European Funds for Regional Economical Development for their financial support to this project. 


\section{References}

[1] M.P.B. Musculus, P.C. Miles, L.M. Pickett, Prog. Energy Combust. Sci. 39 (2013) 246-283.

[2] F. Battin-Leclerc, Prog. Energy Combust. Sci. 34 (2008) 440-498.

[3] Y. Ju, W. Sun, Combust. Flame 162 (2015) 529-532.

[4] W. Sun, S.H. Won, Y. Ju, Combust. Flame 161 (2014) 2054-2063.

[5] S. Nagaraja, W. Sun, V. Yang, Proc. Combust. Inst. 35 (2014) 3497-3504.

[6] P. Dagaut, M. Reuillon, M. Cathonnet, Combust. Flame 101 (1995) 132-140.

[7] O. Herbinet, B. Husson, Z. Serinyel, M. Cord, V. Warth, R. Fournet, P.-A. Glaude, B. Sirjean, F. Battin-Leclerc, Z. Wang, M. Xie, Z. Cheng, F. Qi, Combust. Flame 159 (2012) 3455-3471.

[8] H.K. Ciezki, G. Adomeit, Combust. Flame 93 (1993) 421-433.

[9] R. Minetti, M. Carlier, M. Ribaucour, E. Therssen, L.R. Sochet, Combust. Flame 102 (1995) 298309.

[10] D.M.A. Karwat, S.W. Wagnon, M.S. Wooldridge, C.K. Westbrook, Combust. Flame 160 (2013) 2693-2706.

[11] M.A. Boumehdi, S.A. Stepanyan, P. Desgroux, G. Vanhove, S.M. Starikovskaia, Combust. Flame 162 (2015) 1336-1349.

[12] Y. Yu, G. Vanhove, J.F. Griffiths, S. De Ferrières, J.F. Pauwels, Energy Fuels 27 (2013) 39883996.

[13] S.A. Stepanyan, A.Y. Starikovskiy, N.A. Popov, S.M. Starikovskaia, Plasma Sources Sci. Technol. 23 (2014) 045003.

[14] D. Lee, S. Hochgreb, Combust. Flame 114 (1998) 531-545.

[15] G. Vanhove, G. Petit, R. Minetti, Combust. Flame 145 (2006) 521-532.

[16] C.O. Laux, in:, Phys.-Chem. Model. High Enthalpy Plasma Flows, D. Fletcher, J.-M.

Charbonnier, G.S.R. Sarma, and T. Magin, Rhode-Saint-Genèse, Belgium, 2002. 
[17] M. Crochet, R. Minetti, M. Ribaucour, G. Vanhove, Combust. Flame 157 (2010) 2078-2085.

[18] G. Mittal, M.P. Raju, C.-J. Sung, Fuel 94 (2012) 409 - 417. 